This report was prepared as an account of work sponsored by an agency of the United States Government. Neither the United States Government nor any agency thereof, nor any of their employees, makes any warranty. express or implied, or assumes any legal liability or responsibility for the accuracy, completeness, or usefulness of any information, apparatus, product, or prosess disclosed, or represents that its use would not infringe privately owned rights. Reference herein to any specific commercial product, process, or service by trade name, trademark, manufacturer, or otherwise does not necessarily constitute or imply its endorsement, recommendation, or favoring by the United States Government or any agency thereof. The views and opinions of authors expressed herein do not necessarily state or reflect those of the United States Government or any agency thereof.

\author{
FEB $05: 991$
}

*Presented by J. Labrenz at Lattice '90, International Symposium on Lattice Gauge Theory, Tallahassee, FL, October 8-12, 1990.

** Address after January 1, 1991: Department of Physics, Washington University, St. Louis, MO 63130.

This manuscript has been authored under contract number DE-ACO2-76CH00016 with the U.S. Department of Energy. Accordingly, the U.S. Government retains a non-exclusive, royalty-free license to publish or reproduce the published form of this contribution, or allow others to do so, for U.S. Government purposes. 


\title{
DECAY CONSTANTS and WAVE FUNCTIONS of HEAVY-LIGHT PSEUDOSCALARS *
}

\author{
Claude BERNARD, ${ }^{a \dagger}$ James LABRENZ, ${ }^{b}$ and Amarjit SONI ${ }^{b, a}$ \\ ${ }^{a}$ Institute of Theoretical Physics, Santa Barbara, CA 93106, USA \\ ${ }^{6}$ Physics Department, Brookhaven Nationa' Laboratory, Upton, NY 11973, USA
}

Calculations of the pseudoscalar decay constant for mesons containing one heavy quark (i.e., $f_{D}, f_{B}$ ) are reported. Results at $\beta=6.0$ using the static-quark approximation for the heavy-quark propagator are compared to results using a propagating heavy quark with Wilson fermions at $(\beta=6.0, \beta=6.1$ and $\beta=6.3)$. Further investigation into the dynamics governing these systems is made by measuring the Bethe-Salpeter wave function for each case.

\section{INTRODUCTION}

Matrix elements of pseudoscalar mesons $P$, containing one heavy quark $(Q=c, b$; and one light quark ( $q=u, d, s)$, are of great phenomenological interest and have recently been the subject of a variety of calculational efforts on the lattice. $^{1-4}$ Here, the major obstacle to a successful treatment of such "heavy-light" physics is clear: the existence of a heavy quark (mass $m_{Q}$ ) as a dynamical degree of freedom restricts the grid spacing $a$ to a value $a \ll m_{Q}^{-1}$ so that maintaining a physical dimension $N a$ comparable to typical hadronic scales quickly exhausts current computing capabilities.

There have been two approaches to circumvent this problem. The first, which we will refer to as the "conventional" technique, ${ }^{1}$ is to simulate both heavy and light quarks as standard lattice (Wilson) fermions and to measure some physical quantity, such as $f_{P}$, as a function of $M_{P}$ while increasing the mass of the heavy quark. Clearly at some point in this process, the error induced by large $a M$ effects becomes intolerable, and one must introduce some extrapolation procedure in order to reach physics at higher mass scales. At what value of $a M_{P}$ direct simulation becomes unreliable and extrapolation is necossary is in general a numerical question to which one must address by repeating the calculation at a weaker coupling and checking for scaling behavior.

The second approach to the heavy-light problem on the lattice was initially proposed by Eichten, ${ }^{3}$ and by Lepage and Thacker, ${ }^{5}$ and is commonly referred to as the static-quark technique. Here, one removes the heavy-quark mass as a dynamical scale in the problem by retaining only the leading term in an expansion of the heavy-quark Lagrangian in powers of $1 / m_{Q}$.

We report here recent results for calculations of $f_{P}$, using both methods outlined above. The expected behavior at very large mass scales,

$$
f_{P} \sqrt{M_{P}} \sim \text { constant (up to logs) }
$$

is implicit in the static approximation and may be checked for in conventional calculations. As the estimate of $f_{B}$ made previously using the conventional technique is in poor agreement with the results in the static limit, we report, in Section 4, measurements of the Bethe-Salpeter wave functions as a way to study the difference between the "static-light" and the conventional heavy-light mesons. The lattices used in the calculations are listed in Table 1.

\section{D MESONS AND BEYOND}

We discuss now the heavy-light resuits from the conventional method where "light" is defined to be in the vicinity of the strange-quark mass.

*Presented by J. Labrenz at the International Symposium Lattice '90, Tallahassee, Fl., Oct., 1990.

${ }^{\dagger}$ Address after Jan 1,1991: Dept. of Physics, Washington University, St. Louis, MO 63130.

${ }^{\ddagger} \mathrm{BNL}$ is operated by Associated Universities, Inc. under contract with the U.S. Department of Energy. 


\begin{tabular}{|c|c|c|c|c|c|}
\hline name & $\beta$ & $\sim a^{-1}(\mathrm{GeV})$ & size & $L(f m)$ & confs \\
\hline$A_{16}$ & 5.7 & 1.0 & $16^{3} \times 25$ & 3.2 & 16 \\
$B_{16}$ & 6.0 & 1.75 & $16^{3} \times 39$ & 1.8 & 19 \\
$B_{94}$ & 6.0 & 1.75 & $24^{3} \times 39$ & 2.7 & 8 \\
$C_{12}$ & 6.1 & 2.0 & $12^{3} \times 33$ & 1.2 & 30 \\
$D_{24}$ & 6.3 & 2.75 & $24^{3} \times 49$ & 1.7 & 4 \\
\hline
\end{tabular}

Table 1: The lattices and nominal parameters used.

Using the strange quark eliminates the complication of an extrapolation in $\kappa_{q}$ to the chiral limit and allows us to examine directly the properties of $f_{P}$ as a function of $M_{P}$ at several different couplings.

In Fig. 1 and Table 2 we re-examine a subset of the results at $\beta=6.1$ (originally presented in Ref. 1) and compare them to recent results at $\beta=6.0$ and preliminary results (from only four configurations) at $\beta=6.3$. Only statistical errors are shown. We draw the following conclusions:

1) As noted in Ref. 1, the data beyond the mass of the $D$ meson appear consistent with the expected large-mass scaling law given by (1).

2) The suspicion that such behavior could be a lattice artifact due to the largeness of $a M$ is so far unsubstantiatter, as the general shape in Fig. 1 appears to be scaling over the range of couplings tested.

The second conclusion is clearly highly preliminary, as only a fraction of the available data set at $\beta=6.3$ has been analyzed so far.

\section{THE STATIC-QUARK APPROXIMATION}

We assume that the reader is familiar with the static-quark approximation on the lattice 3,5 and provide only those details necessary foi the presentation of our numerical results.

\subsection{Calculational Methods}

For the static-quark propagi-ior, we use ${ }^{3}$

$$
\begin{aligned}
& S_{Q}^{\text {iatt }}(x, y)=\delta^{3}(\vec{x}-\vec{y}) e^{-a m_{Q}\left|x_{0}-y_{0}\right|} \\
& \times\left\{\begin{array}{l}
\theta\left(x_{0}-y_{0}\right)\left(\frac{1+\gamma_{0}}{2}\right) \prod_{t=x_{0}-1}^{y_{0}} U_{\hat{j}}^{\dagger}(\vec{y}, t) \\
+\theta\left(y_{0}-x_{0}\right)\left(\frac{1-\gamma_{0}}{2}\right) \prod_{t=x_{0}}^{y_{0}-1} U_{\dot{j}}(\vec{y}, t)
\end{array}\right\} .
\end{aligned}
$$

Given, then, the standard definitions

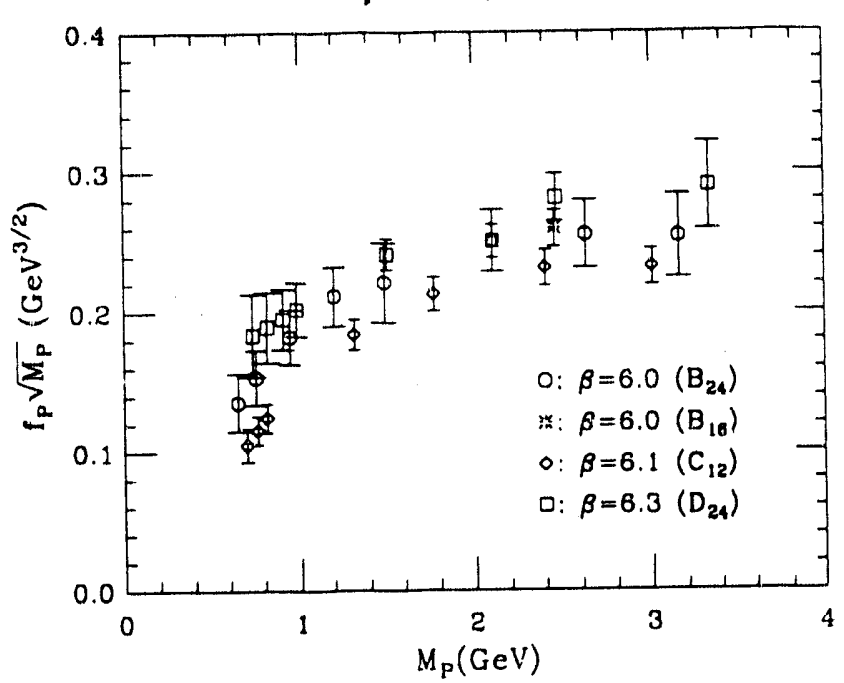

Figure 1: The decay constant of "heavy-strange" mesons from the conventional method. See also Table 2.

\begin{tabular}{|c|c|c|c|c|c|}
\hline name & $\beta$ & $\kappa_{q}$ & $\kappa_{Q}$ & $a M_{P}$ & $a^{3 / 2} f \sqrt{\Lambda 1}$ \\
\hline \hline$B_{24}$ & 6.0 & .154 & .154 & $0.373(70)$ & $.059(09)$ \\
& & & .135 & $0.852(11)$ & $.095(12)$ \\
& & & .088 & $1.805(17)$ & $.110(13)$ \\
\hline$C_{12}$ & 6.1 & $“ .152 ”$ & $“ .152 "$ & $0.353(28)$ & $.0371(42)$ \\
& & & .130 & $0.887(15)$ & $.0752(43)$ \\
& & & .100 & $1.504(14)$ & $.0820(45)$ \\
\hline$D_{24}$ & 6.3 & .150 & .150 & $0.268(07)$ & $.0402(64)$ \\
& & & .140 & $0.547(05)$ & $.0529(25)$ \\
& & & .110 & $1.211(12)$ & $.0636(68)$ \\
\hline
\end{tabular}

Table 2: A subset of the data plotted in Fig. 1. Note that $\kappa_{q}=$ ".152" is a linear interpolation between $\kappa_{q}=.153$ and $\kappa_{q}=.151$.

$$
\begin{aligned}
A_{\mu} & =\bar{\psi}_{Q} \gamma_{\mu} \gamma_{5} \psi_{q}^{\prime}, \quad \text { and } \\
\left\langle 0\left|A_{\mu}(x)\right| P(\vec{p})\right\rangle & =-i f_{P} p_{\mu} e^{-i p \cdot x},
\end{aligned}
$$

the calculation of the decay constant in the static limit follows from a conventional computation of the axial-axial Green function, with the substitution of (2) for the heavy-quark propagator. In practice, however, such a computation is highly contaminated with contributions from excited states, ${ }^{6}$ as illustrated in Fig.2.

The calculation is made feasible through the use of "smeared" operators to enhance the overlap with the ground state (see Fig. 2) and gauge fixing to extract gauge-invariant physics from these gauge-non-invariant quantities. Define the smeared axial current as

$$
A_{\mu}^{s}(x) \equiv \frac{1}{n^{3}} \int_{V_{n}} d^{3} y \bar{\psi}_{q}(x) \gamma_{\mu} \gamma_{5} \psi_{Q}(x+\vec{y}) .
$$


The "source-smeared" correlator then reduces to

$$
\begin{aligned}
& \left\langle A_{0} A_{0}^{s}\right\rangle^{\text {latt }} \\
& \quad=\left\langle\frac{\operatorname{Tr}}{n^{3}} \sum_{i}^{(n)}\left[\frac{1 \pm \gamma_{0}}{2} P^{ \pm} S_{q}\left(\vec{\imath}, t_{x} ; \vec{o}, t_{o}\right)\right]\right\rangle \\
& \quad \longrightarrow \zeta_{s} e^{-a\left(M_{P}-m_{Q}\right)\left|t_{x}-l_{0}\right|},
\end{aligned}
$$

Where $P^{ \pm}$stands for the appropriate product of links in (2). The source/sink-smeared correlator $\left\langle A_{0}^{s} A_{0}^{s}\right\rangle^{\text {latt }}$ is similarly defined; ${ }^{7}$ its residue is denoted $\zeta_{s s}$. In terms of these quantities, the decay constant is given by

$$
f_{P} \sqrt{M_{P}}=Z_{A}^{s t a t} \sqrt{2 \kappa_{q}} \sqrt{\frac{2 \zeta_{s}^{2}}{\zeta_{s s}}} \times a^{-3 / 2},
$$

where $Z_{A}^{\text {stat }}$ is the perturbatively-calculated renormalization constant for the axial-vector current. ${ }^{8}$

Fits to a single exponential were done on the quantities $\left\langle A_{0} A_{0}^{s}\right\rangle^{\text {latt }}$ and $\left\langle A_{0}^{s} A_{0}^{s}\right\rangle^{\text {latt }}$ using singleelimination subsets of configurations; the best fits were then used with (8) and the standard jackknifing procedure to give our estimates of the statistical error in the computation.

\subsection{Numerical Results}

The decay constant was computed using the static approximation on lattices $A_{16}$ and $B_{24}$, fixed to Landau gauge. The results from lattice $B_{24}$, quoted as our best values for this method, are shown in Fig. 3 ; lattice $A_{16}$ gives a consistent lower bound, but the result is unstable to the choice of fitting interval.

A comparison of Figs. 1 and 3 reveals the magnitude of the discrepancy between the static and conventional results. Note that the quantity in Fig. 3 is a bare lattice quantity; its perturbative renormalization contains an uncertainty stcmming from the choice of coupling but has been estimated to be $8.9 Z_{A}^{\text {stat }} \approx 0.6-0.8$. The inclusion of this factor improves the situation but falls short of a resolution. There are then several possible explanations for the discrepancy:

- First, we may still be at values of $a M$ that are too large to produce reliable results in the conventional method. Completion of the calculation at $\beta=6.3$ should shed light on this question.

- Secondly, $\mathcal{O}(a)$ corrections may be significant. This issue we again defer to "computations in progress," which will use an improved action. ${ }^{10}$

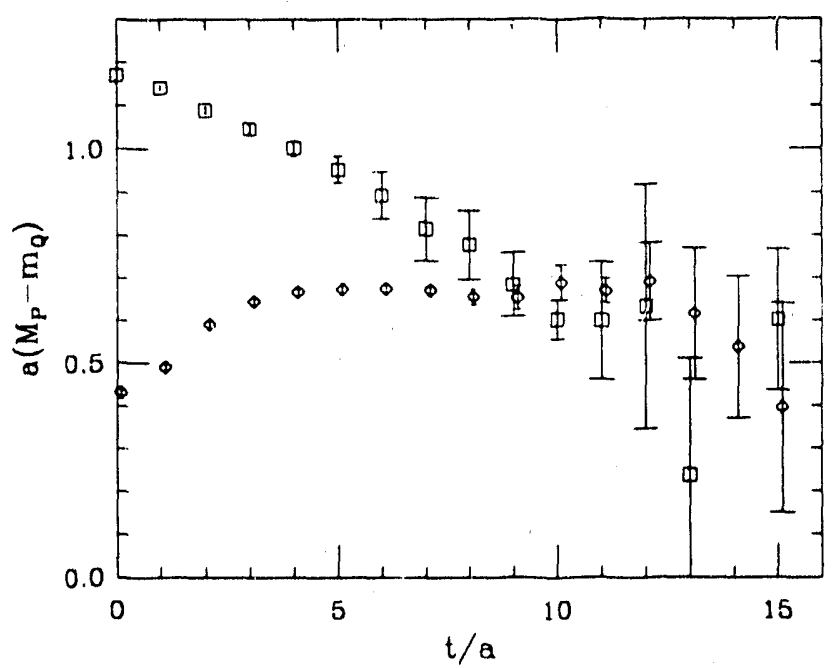

Figure 2: The "binding energy" from local (ㅁ) and source-smeared $(\diamond)$ correlators in the static approximation, from lattice $B_{24}$, with $\kappa_{q}=.154$.

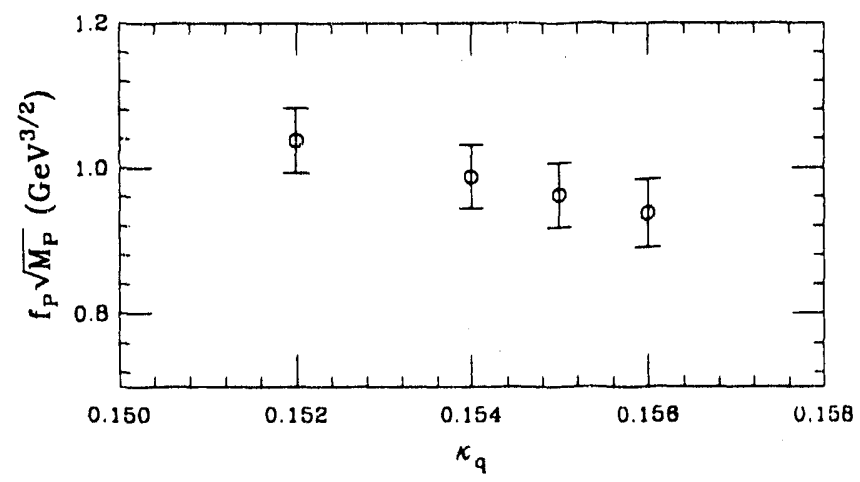

Figure 3: The decay constant in the static limit at $\beta=6.0$ (lattice $B_{24}$ ), before renormalization by $Z_{A}^{s t a t}$.

- Thirdly, the fact that $Z_{4}^{\text {stat }}$ is calculated to be such a large "correction" may be an indication that some non-perturbative method of renormalization is required.

- Finally, there exists the possibility that the static-quark results may be altered in the mass region below and including the $\mathrm{B}$ meson by the inclusion of $\left(1 / m_{Q}\right)$ terms. However, this, by itself, cannot explain the discrepancy, since the conventional results seem to show asymptotic behavior (no large $\left(1 / m_{Q}\right)$ corrections) at a value of $f \sqrt{M}$ that is inconsistent with the static approximation.

Thus, we proceed to investigate the normalization of the static-quark measurements in the following section.

\section{WAVE FUNCTIONS}

\subsection{Motivation}

The Bethe-Salpeter wave functions (BSWF) of SU(3) hadronic states were first computed on the lattice by Gottlieb. ${ }^{11}$ They are of interest in the 
present context of the heavy-light system primarily for two reasons. First, as was pointed out (1) us by Eichten, 12 the wave function may provile a quantitative check on the decay constant calculations: for highly non-relativistic bound states, the BSWF reduces to the ordinary quantummechanical wave function, whose value at the origin is then determined by properly normalizing the measurement. For such states, the decay constant is given by (again, up to logs)

$$
f_{P} \sqrt{M_{P}}=2 \sqrt{3}\left|\phi_{P}(0)\right| .
$$

The validity of the approximation here (one is requiring the light quark to be non-relativistic) must, however, be checked empirically. Secundly, wave functions may provide useful qualitative information-e.g., the approximate physical size of the states, how this size compares to the parameters of the computation, and how the states differ under the two methods.

\subsection{The Calculation}

We define, in addition to the local pseudoscalar current $J_{5}(x)$, the non-local current

$$
J_{5}(x, \vec{r}) \equiv \bar{\psi}_{Q}(x+\vec{r}) \gamma_{5} \psi_{q}(x) .
$$

The BSWF, defined in a fixed gauge ${ }^{11}$ as

$$
\Phi_{P}(\vec{r}) \equiv\left\langle 0\left|J_{5}(x, \vec{r})\right| P\right\rangle
$$

is extracted from a lattice calculation of the twopoint correlator, defined by

$$
\Gamma(\vec{r}, t) \equiv \sum_{\vec{x}}\left\langle 0\left|\hat{\mathcal{T}} J_{5}(x, \vec{r}) J_{5}^{\dagger}(0)\right| 0\right\rangle
$$

via the large-time relation

$$
\frac{\Gamma(\vec{r}, t)}{\Gamma(\overrightarrow{0}, t)} \longrightarrow \frac{\Phi_{P}(\vec{r})}{\Phi_{P}(\overrightarrow{0})} \equiv \Psi_{P}(\vec{r}) .
$$

We average the quantity $\Gamma(\vec{r}, t)$ over all points symmetric uncior cubic rotations. In the static approximation, $\Psi_{P}$ is computed using a smeared current, $J_{5}^{s}$ (analogous to (5)), in place of the local current at the origin of the correlation function (12). Our discussion here is limited to Landau gauge. ${ }^{13}$ Errors are again computed using the jackknife tochnique and are purely statistical.

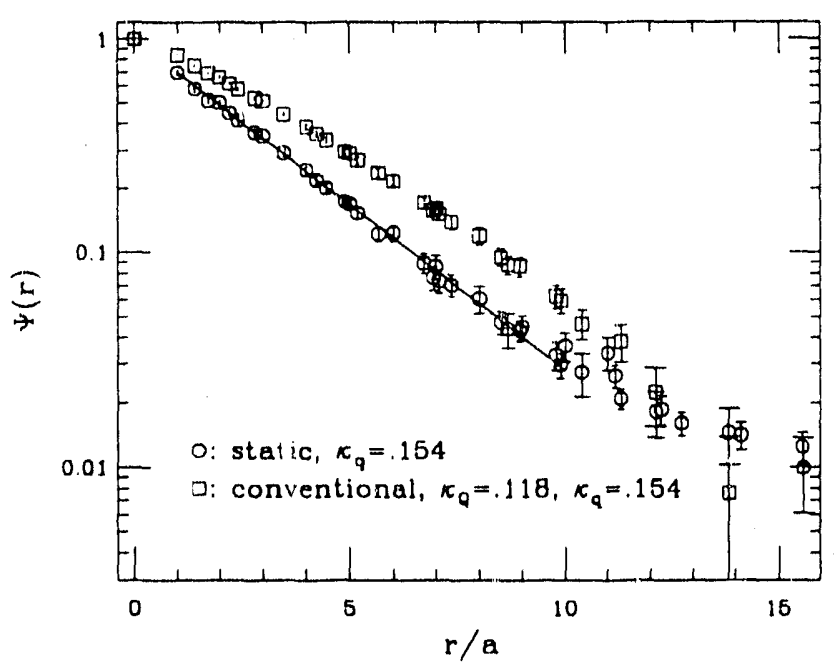

Figure 4: The Landau-gauge wave functions, as given by eq. 1 , from lattice $B_{24}$, with $\kappa_{q}=.154$.

\subsection{Results}

In Fig. 4 are shown the results of the wave function measurements at $\beta=6.0$ using both the conventional and static-quark techniques, with a "light" quark of $\kappa_{q}=.154$. The qualitative behavior of the two states is quite different: the staticlight wave functions in general fall more quickly than the conventional ones, and empirically fit well to a simple exponential (such as that produced by a Coulombic potential).

However, independent of such observations, let us momentarily assume non-relativistic behavior, integrate the radial distribution $r^{2}|\Psi(r)|^{2}$, and compute the decay constant via (9). For clarity, we enumerate the expanded range of possible techniques:

1a. conventional, direct (as in Fig. 1),

1b. conventional, wave function (from eq. 9),

2a. static, direct (as in Fig. 3), and

2 b. static, wave function.

For the static case (using "light" quarks of $\kappa_{q}=.155, .154$ and .152$)$, we find close agreement between the bare lattice results. $\Lambda \mathrm{t} \kappa_{q}=.154$, for example, method 2a. gives $a^{3 / 2} f \sqrt{M}=.43(2)$, whereas method 2b. gives $a^{3 / 2} f \sqrt{M}=.41(2)$. Note, however, that such precise agreement is certainly fortuitous - the (large and uncertain) renormalization constants have not been included.

Moreover, any numerical agreement in the check described above may in fact be very misleading. In Fig. 5 we plot the ratio of bare decay 


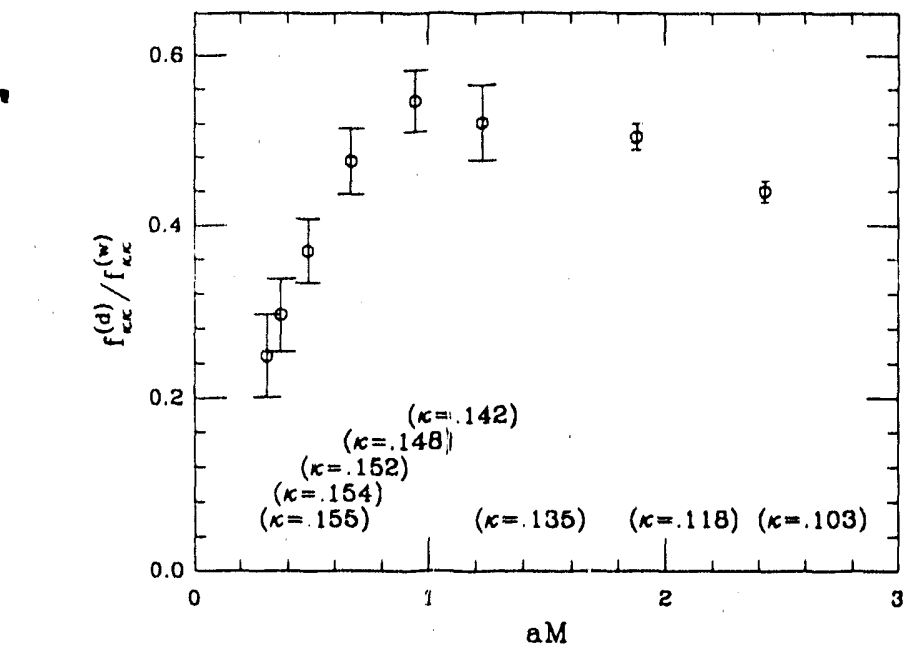

Figure 5: The ratio of degenerate-meson, bare decayconstants, $\mathrm{f}$ (direct)/f(wave function), from lattice $B_{24}$.

constants from methods $1 \mathrm{a}$. and $1 \mathrm{~b}$. for degenerate (instead of heavy-light) mesons. In the lowmass region of the plot, we interpret the smallness of this ratio as evidence of a relativistic bound state-the wave-function technique gives absurdly large results. ${ }^{14,15}$ Despite the difference in reduced mass between these degenerate systems and the corresponding static-light systems, it thus seems unlikely that the light quarks of the latter are non-relativistic. Further, one can calculate the mean velocity of the light quark directly from the wave function under the non-relativistic assumption. For the static-light wave function in Fig. 4, for example, we find $v / c \approx 1$, in violation of the assumption. Thus, for the light-quark masses used so far, the static method cannot be realistically tested by the wave-function approach.

\section{CONCLUSIONS}

In general, our results indicate that the staticquark and conventional methods of computing heavy-light physics are producing highly inconsistent answers for the decay constant calculation. Thus we feel that it is still premature to base an estimate of $f_{B}$ on the static results. However, we are proceeding to clarify the discrepancies by 1) completing the computation at $\beta=6.3$ with at least fifteen configurations, 2) further studying the wave functions for a wide variety of cases, and 3 ) including the $1 / m_{Q}$ and $\mathcal{O}(a)$ corrections into the existing calculations.

\section{ACKNOWLEDGEMENTS}

We thank M. Creutz, E. Eichten, B. Hill, P. Mackenzie, C. Sachrajda and S. Sharpe for useful discussions. The computing was done at the $\mathrm{Na}$ tional Energy Research Supercomputer Center in part under the "Grand Challenge" program and at the San Diego Supercomputer Center.

\section{REFERENCES}

1. C. Bernard, T. Draper, G. Hockney, and A. Soni, Phys. Rev. D38 (1988) 3540.

2. M. B. Gavela, et al., Phys. Lett. 206B (1988) 113; T. A. DeGrand and R. D. Loft, Phys Rev D 38 (1988) 954.

3. E. Eichten, Nucl. Phys. B (Proc. Suppl.) 4 (1988) 170.

4. E. Eichten, et al., Nucl. Phys. B (Proc. Suppl.) 17 (1990) 529; C. Allton, et al., Rome preprint 747 (July, 1990); C. Alexandrou, et al., Wuppertal preprint B 90-18 (1990).

5. G. P. Lepage and B. A. Thacker, Nucl. Phys. B (Proc. Suppl.) 4 (1988) 199.

6. Ph. Boucaud, et al., Phys. Lett. 220B (1989) 219.

7. The non-local operator $A_{0}^{s}$ used at the sink in the source/sink- smeared correlator is in fact not that given by eq. 5 , but the analogous operator with the "smearing" performed over the light-quark d.o.f. This ensures that $S_{q}$ from only a single source point enters the computation. The desired correlator is equivalent to the one computed by translation invariance.

8. E. Eichten and B. Hill, Phys. Lett. B 234 (1090) 511 , and Phys. Lett. B 243 (1990) 427; Ph. Boucaud, et al., Phys. Rev. D 40 (1989) 1529.

9. P. Mackenzie, these proceedings.

10. G. Heatlie, et al., Rome preprint 737 (April, 1990).

11. S. Gottlieb, Proceedings of the Tallahassee Lattice Gauge Theory Conference, D. Duke and G. Owens, eds., World Scientific, Singapore (1985) 105.

12. E. Eichten, private communication; see also H. B. Thacker, these proceedings.

13. At $\beta=5.7$ we have made some preliminary measurements of Coulomb-gauge, static-light wave functions $\left(\kappa_{q}=.155, .160\right)$. We find $|\phi(0)|^{C o u l} \approx 1.3|\phi(0)|^{L a n}$.

14. A qualitatively similar result for $f \sqrt{M}$ via method $1 \mathrm{~b}$. is obtained from staggered-fermion wave functions computed by Gupta et al. See, for example, S. Sharpe, University of Washington preprint, 40423-10 P90 (1990).

15. We note that even in the moderate mass region $\left(\kappa_{q}=\right.$ $.148-.135$ ), the ratio in Fig.5 does not approach 1. Possible causes for this behavior-such as the absence here of renormalization factors, or the onset of large $a M$ effects-are under investigation. 

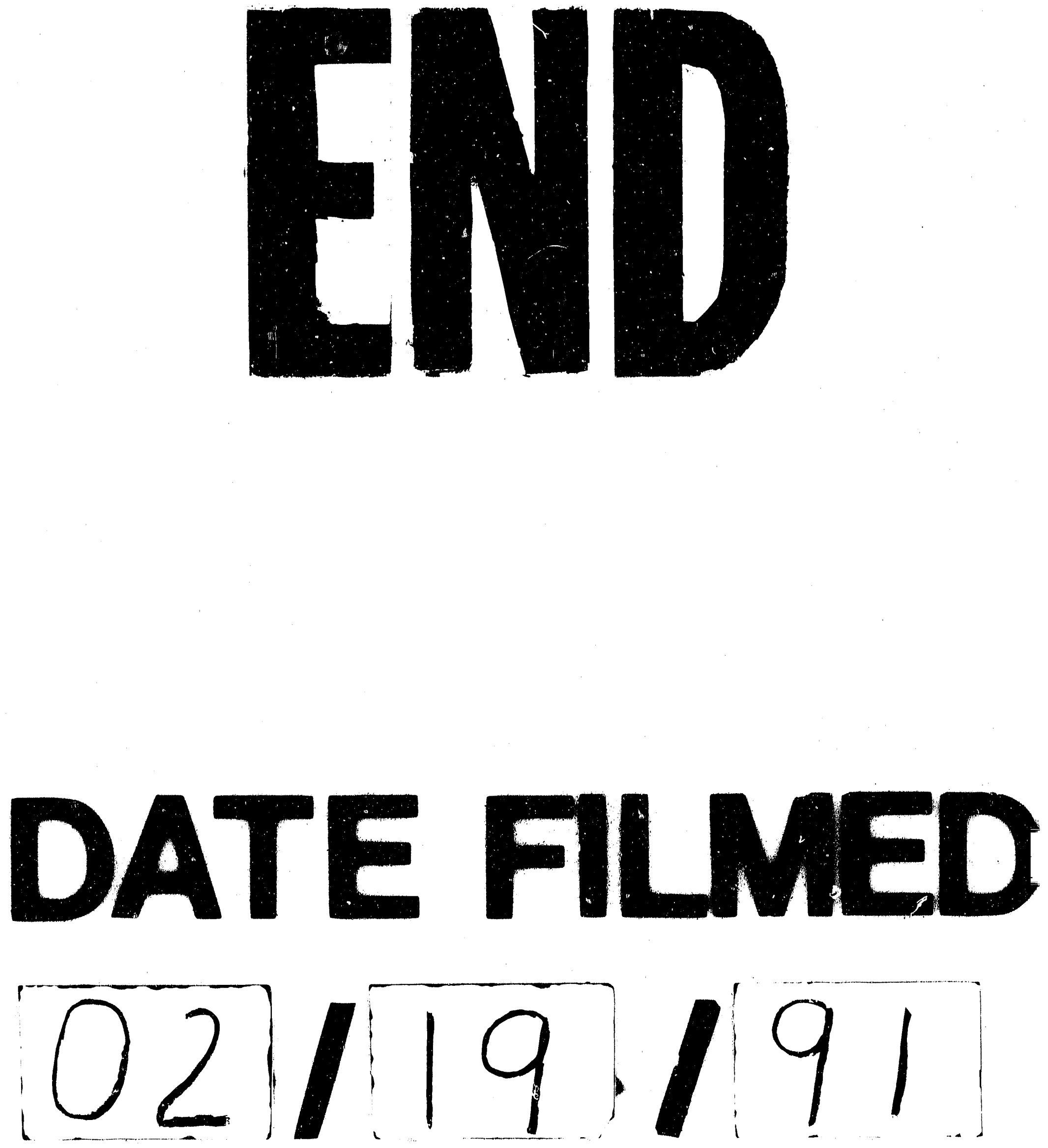
